\title{
Lobinger, Katharina (Hrsg.): Handbuch Visuelle Kommunikationsforschung
}

\author{
Wiesbaden: Springer VS 2019. 749 Seiten. Preis: $€$ 69,99 (e-book)
}

\section{Daria Gordeeva}

Online publiziert: 25. September 2020

(C) Der/die Autor(en) 2020

Bilder sind allgegenwärtig. Sie durchdringen unseren Alltag, konstruieren Wirklichkeit und sind aus der modernen mediatisierten Gesellschaft nicht mehr wegzudenken. Der Erforschung von Medienbildern und visueller Kommunikation widmet sich das vorliegende Handbuch, das die Kommunikationswissenschaftlerin Katharina Lobinger als Herausgeberin verantwortet.

Der knapp 750-seitige Sammelband gewährt einen umfassenden Einblick in die visuelle Kommunikationsforschung. Dabei handelt es sich um ein noch relativ junges Forschungsfeld, das seine „offizielle Institutionalisierung“ mit der Gründung der Fachgruppe Visuelle Kommunikation in der Deutschen Gesellschaft für Publizistikund Kommunikationswissenschaft (DGPuK) im Jahr 2000 erfuhr. Das kurz vor deren 20. Geburtstag erschienene Handbuch führt die Aktivitäten der Fachgruppe zusammen und reiht sich hiermit ein in die Tradition, die Verhandlungen auf den Jahrestagungen in Sammelbänden zu publizieren. Das Werk zielt darauf ab, ,den aktuellen Forschungs- und Diskussionsstand“ zu bündeln und dieses dynamische und verstreut publizierende Forschungsfeld innerhalb der Kommunikations- und Medienwissenschaft sichtbar zu machen.

Das Handbuch versammelt 33 Beiträge, die sich, wie auch das Forschungsfeld selbst, durch , die Bandbreite unterschiedlicher theoretischer, methodischer und inhaltlicher Zugänge“" auszeichnen (S. 2-3). Im einleitenden Beitrag grenzen Katharina Lobinger und Rebecca Venema die visuelle Kommunikationsforschung von der allgemeinen Bildwissenschaft ab und betonen ihre trans- und interdisziplinäre Ausrichtung, die sich auch in der fachübergreifenden Zusammensetzung des AutorInnenteams zeigt. Katharina Lobinger trägt der Vielfalt und Komplexität des For-

D. Gordeeva, M.A. ( $\bowtie)$

Institut für Kommunikationswissenschaft und Medienforschung, Ludwig-Maximilians-Universität München, Oettingenstraße 67, 80538 München, Deutschland

E-Mail: Daria.Gordeeva@ifkw.Imu.de 
schungsfeldes Rechnung und bündelt die vielfältigen Inhalte in vier Abschnitte: Theoretische Grundlagen, Themenfelder, Methoden und Herausforderungen der Visuellen Kommunikationsforschung.

Der erste Teil des Handbuchs liefert einen theoretischen Rahmen für die Analyse visueller Medien und rüstet Leserinnen und Leser mit Grundbegriffen aus. Beiträge in diesem Abschnitt diskutieren semiotische und handlungsorientierte Zugänge zur Erforschung alltäglicher Bildpraktiken und erörtern gesellschaftliche Rahmenbedingungen, die sich mit den Schlagwörtern Digitalisierung und Mediatisierung beschreiben lassen. In den Beiträgen zum Emotionalisierungspotenzial von Bildern, der visuellen Erinnerungskultur und der Authentizitätsinszenierung werden weitere zentrale Facetten visueller Kommunikation aufgegriffen.

Im zweiten und umfangreichsten Abschnitt finden sich 16 Beiträge zu Forschungsschwerpunkten. Neben den „klassischen“ Feldern des Fotojournalismus, Terror- und Konfliktberichterstattung, der politischen Kommunikation, der Werbung und der Public Relations finden auch ,innovative“ Themen Platz, die im Zuge der Digitalisierung an Bedeutung gewonnen und ,bisher noch vergleichsweise wenig wissenschaftliche Aufmerksamkeit erfahren haben“"(S. 7). So rücken Beiträge die Wissenschaftskommunikation und Datenvisualisierung sowie die Alltagskommunikation (Selbstinszenierung, digitales Storytelling und Photo Sharing) in den Untersuchungsfokus. Karikaturen und Comics, Geschlechter- und Körperbilder, Altersstereotype und Geomedien runden das umfassende Themenspektrum ab.

Das dritte Kapitel beschreibt methodische Zugänge zur visuellen Kommunikationsforschung. Das Handbuch verzichtet auf eine systematische Darstellung einzelner Forschungsmethoden, verdeutlicht aber die Eigenheiten der Medienbildanalyse anhand von Praxisbeispielen und stellt ein Analyseinstrumentarium zur Verfügung. Dabei werden Bilder einerseits als (historische) Quellen und Untersuchungsobjekte begriffen, die stets in ihren Produktions- und Rezeptionskontexten analysiert werden sollen. Andererseits wird erläutert, wie visuelle Medien auch im Forschungsprozess gewinnbringend eingesetzt werden können, zum Beispiel als Gesprächsstimuli in Interviews oder zur Visualisierung komplexer Sachverhalte.

Der vierte und letzte Teil befasst sich mit den Herausforderungen thematischer, methodologischer, forschungsethischer und juristischer Art, zum Beispiel der Multimodalität der Medienkommunikation, den rechtlichen Rahmenbedingungen der Bildverwendung und der Bildethik. Der Abschlussbeitrag schlägt eine Brücke in die Zukunft visueller Kommunikationsforschung. Plädiert wird unter anderem für eine intensivere Beschäftigung mit „Akteursverflechtungen“ (S. 727), ,komplexen Seh- und Sichtbarkeitsrelationen und den in ihnen sedimentierten Macht- und Herrschaftsverhältnissen“ (S. 745), für die Forschung im Bereich Augmented und Virtual Reality sowie die verstärkte Einbindung visueller Komponenten in akademische Publikationen.

In diesem breiten Themen- und Methodenrepertoire scheint allerdings das Medium Film ein blinder Fleck zu sein. Zwar wird die prägende Rolle der Filme für die Erinnerungskultur, Kriegsdarstellung, Wissenschaftskommunikation, das digitale Geschichtserzählen oder die Stereotypisierung in einzelnen Beiträgen angedeutet. Dennoch fokussiert sich die bisherige visuelle Kommunikationsforschung im Fach primär auf unbewegte Medienbilder, sogenannte „still images“ (S. 127). Wün- 
schenswert wäre eine stärkere Integration von Film- und Fernsehbildern in dieses dynamische, zukunftsorientierte und offene Forschungsfeld.

Dem im Vorwort formulierten Anspruch, interessierten Forscherinnen und Forschern einen einfachen Einstieg in die Untersuchung visueller Kommunikation zu ermöglichen, werden die Herausgeberin, Autorinnen und Autoren gerecht. Man kann deshalb vermuten, dass das Handbuch eine breite und intensive Rezeption erfahren wird. Eine durchdachte Strukturierung, Beispiele aus der Forschungspraxis sowie knappe Zusammenfassungen der Beiträge erleichtern das Verständnis. Besonders positiv hervorzuheben ist die ansprechende Gestaltung des Sammelbandes: mit vielen Bildern, Grafiken und Tabellen. Katharina Lobinger gelingt ein facettenreiches Überblickswerk, das ein komplexes wie spannendes Forschungsfeld eindrucksvoll ausleuchtet.

Funding Open Access funding enabled and organized by Projekt DEAL.

Open Access Dieser Artikel wird unter der Creative Commons Namensnennung 4.0 International Lizenz veröffentlicht, welche die Nutzung, Vervielfältigung, Bearbeitung, Verbreitung und Wiedergabe in jeglichem Medium und Format erlaubt, sofern Sie den/die ursprünglichen Autor(en) und die Quelle ordnungsgemäß nennen, einen Link zur Creative Commons Lizenz beifügen und angeben, ob Änderungen vorgenommen wurden.

Die in diesem Artikel enthaltenen Bilder und sonstiges Drittmaterial unterliegen ebenfalls der genannten Creative Commons Lizenz, sofern sich aus der Abbildungslegende nichts anderes ergibt. Sofern das betreffende Material nicht unter der genannten Creative Commons Lizenz steht und die betreffende Handlung nicht nach gesetzlichen Vorschriften erlaubt ist, ist für die oben aufgeführten Weiterverwendungen des Materials die Einwilligung des jeweiligen Rechteinhabers einzuholen.

Weitere Details zur Lizenz entnehmen Sie bitte der Lizenzinformation auf http://creativecommons.org/ licenses/by/4.0/deed.de.

Daria Gordeeva ist Doktorandin und wissenschaftliche Mitarbeiterin am Institut für Kommunikationswissenschaft und Medienforschung der Ludwig-Maximilians-Universität München. 